Revista de Matemática: Teoría y Aplicaciones 2009 16(1) : 188-198

CIMPA - UCR ISSN: 1409-2433

\title{
UN PROCEDIMIENTO DE FUERTE REDUCCIÓN DE LAS DIMENSIONES DEL RCPS/ $\pi$
}

\author{
Ramón Álvarez-Valdés Olaguíbel* ${ }^{*}$ Enric Crespo ${ }^{\dagger}$ \\ José Manuel Tamarit Goerlich ${ }^{\ddagger}$ M. Fulgencia Villa Juliá ${ }^{\S}$
}

Recibido/Received: 20 Feb 2008 - Aceptado/Accepted: 14 Dic 2008

\begin{abstract}
Resumen
En el campo de la secuenciación de proyectos se ha introducido recientemente el concepto de recursos parcialmente renovables. Desde el punto de vista teórico, pueden considerarse como una generalización de los renovables y los no renovables. Desde el práctico, permiten ampliar la modelización a situaciones que no eran posibles anteriormente, especialmente en los campos de los horarios y las situaciones laborales. Además, este problema, como tantos que modelizan procesos reales, adquiere rápidamente unas dimensiones que son difíciles de tratar directamente. Por ello, aquí nos centramos en la exposición de un potente preproceso que reduce notablemente la dimensión de los problema y mejora la eficiencia de los métodos de solución posteriormente empleados que, por razones de espacio, se describen en otro trabajo. Se muestran los resultados del preproceso utilizado con diferentes instancias existentes en la literatura donde se consiguen excelentes resultados.
\end{abstract}

Palabras clave: Secuenciación de proyectos, Preproceso, Recursos Parcialmente Renovables.

\footnotetext{
Abstract

Recently, in the field of project scheduling problems the concept of partially renewable resources has been introduced. Theoretically, it is a generalization of both

${ }^{*}$ Dep. d'Estadística i Investigació Operativa. Universitat de València, València, España. E-Mail: ramon.alvarez@uv.es.

${ }^{\dagger}$ Departament de Matemàtiques per a l'Economia i l'Empresa, Facultat d'Economia, Universitat de València, Avgda. dels Tarongers, s/n. 46071-València, España. E-Mail: enric.crespo@uv.es.

${ }^{\ddagger}$ Misma dirección que R. Alvarez. E-Mail: jose.tamarit@uv.es.

${ }^{\S}$ Dep. de Estadística e Investigación Operativa Aplicada y de Calidad. Universidad Politécnica de Valencia, Valencia, España. E-Mail: mfuvilju@eio.upv.es.
} 
renewable and non-renewable resources. From an applied point of view, partially renewable resources allow us to model a large variety of situations that do not fit into classical models, but can be found in real problems in timetabling and labour scheduling. When modelling real problems, the problem of project scheduling with partially renewable resources, as many other combinatorial problems, gets such large dimensions that it is quite difficult to apply solution procedures. In this paper, we describe some powerful preprocessing techniques that reduce significantly the dimension of the problems and therefore improve the efficiency of any algorithm used for solving them. The paper shows the excellent results obtained by these techniques on a set of test instance taken from the literature.

Keywords: Project Scheduling, Preprocessing, Partially Renewable Resources.

Mathematics Subject Classification: 90B35.

\section{Introducción}

La secuenciación de proyectos consiste en determinar el inicio y final de un conjunto de actividades en un proyecto. Dichas actividades están ligadas entre sí por relaciones de precedencia y requieren uno o más recursos. La secuenciación de proyectos ha sido objeto de una gran atención en la investigación desde que los primeros métodos, CPM y PERT, fueron desarrollados en los años 50. Estos procedimientos fueron capaces de resolver grandes problemas y fueron considerados una herramienta muy útil en el proceso de planificación. Sin embargo, ambos presuponen que los recursos son ilimitados con lo que su aplicación queda muy limitada en los problemas reales. Por esto muchos investigadores comenzaron a estudiar el caso de los recursos limitados (RCPSP) del cual se han desarrollado hasta el momento muchos algoritmos exactos y aproximados. El libro de Demeulemeester y Herroelen [4] muestra una excelente descripción de la actual situación de la investigación. El problema clásico del RCPSP incluye dos tipos de recursos; los renovables, cuya disponibilidad se renueva en cada período del intervalo de planificación, y los no renovables, cuya disponibilidad se va reduciendo a lo largo del proyecto a medida que se consumen. Sin embargo estos dos tipos de recursos no son suficientes para representar muchas situaciones reales por lo que han sido propuestos algunos otros tipos de recursos como los comprometidos (allocatable) [6, 10] o los acumulativos [7, 8].

En nuestro trabajo consideramos los recursos parcialmente renovables, introducidos por Böttcher et al. [3] en 1999. La disponibilidad de este tipo de recursos está asociada a un subconjunto de periodos del horizonte de planificación y las actividades sólo consumen el recurso si son procesadas dentro de esos periodos. Este tipo de recursos pueden ser un instrumento poderoso para resolver problemas de secuenciación de actividades. Además, desde el punto de vista teórico, engloban como casos particulares tanto a los renovables como a los no renovables. Por otro lado, los recursos parcialmente renovables permiten modelizar complicadas reglas laborales y restricciones en problemas de horarios como casos concretos de problemas de secuenciación. Como ejemplo consideremos un proyecto que involucra recursos humanos. Podemos encontrarnos con condiciones laborales tales como trabajar a lo sumo dos días del fin de semana cada tres semanas consecutivas. Esta restricción no puede ser modelizada como un recurso renovable porque eso exigiría 
considerar cada periodo por separado. Tampoco puede serlo como uno no renovable porque tendríamos que considerar todo el horizonte de planificación. Sin embargo podemos hacerlo como un recurso parcialmente renovable con un conjunto de periodos $6,7,13,14,20,21$ que incluyen los días de los tres primeros fines de semana y con una disponibilidad de 2 unidades. Cada tarea consume 1 unidad de dicho recurso durante cada día del fin de semana en el que es procesada. Supongamos tres actividades A, B y C secuenciadas en la escala temporal. De ellas, la actividad A está en proceso en los periodos 5,6 y 7 y, por tanto, consume 2 unidades del recurso. La actividad B se realiza en los periodos del 9 al 12, ambos inclusive, y no consume nada. Por fin, la actividad $\mathrm{C}$ comienza en el $16 \mathrm{y}$ acaba en el 20 y consume únicamente 1 unidad en el periodo 20. Sería imposible que estas tres tareas fueran realizadas por el mismo trabajador porque excedería la disponibilidad de recursos.

Böttcher et al.[3] propusieron una formulación entera y desarrollaron algoritmos exactos y heurísticos. Schirmer [9] estudió ampliamente este nuevo tipo de recursos en su libro sobre problemas de secuenciación de actividades. En él presentó muchos ejemplos de condiciones especiales que podían ser adecuadamente modelizadas usando los recursos parcialmente renovables y también presentó varias familias de algoritmos aproximados para resolver el problema de secuenciación de actividades con recursos parcialmente renovables $(\operatorname{RCPSP} / \pi)$. En este trabajo describimos un preproceso que reduce las dimensiones del problema tanto en los recursos como en los tiempos posibles de finalización, a partir de aquí TPF, de las actividades y, por tanto, mejora la eficiencia de los algoritmos.

En el apartado 2 definimos los elementos del problema y exponemos una formulación entera del mismo. El apartado 3 describe los aspectos principales del preproceso. En el 4 exponemos tanto las instancias de la literatura utilizadas para las pruebas como los resultados obtenidos. Por fin, el apartado 5 expone conclusiones y una breve idea sobre los trabajos subsiguientes relacionados que, por razones de espacio, se exponene en otros trabajos.

\section{Formulación del problema}

El RCPSP $/ \pi$ puede ser definido de la manera siguiente: Sea $J$ el conjunto de $n=|J|$ actividades, numeradas de 1 a $n$, donde las actividades ficticias 1 y $n$ representan el inicio y final del proyecto. Sea $P_{j}$ el conjunto de actividades que son predecesoras inmediatas de la actividad $j$ y $P_{j}^{\prime}$ el conjunto de todas las predecesoras de $j$. Cada actividad $j$ tiene una duración $d_{j}$ y no puede ser interrumpida. Sea $R$ el conjunto de recursos parcialmente renovables. Cada recurso $r \in R$ tiene una disponibilidad total $K_{r}$ y un conjunto de periodos asociado $\Pi_{r}$. Una actividad $j$ que necesite del recurso $r$ consumirá $k_{j r}$ unidades del mismo en cada periodo $t \in \Pi_{r}$ en que esté en proceso. Finalmente, sea $T$ el último periodo del horizonte de planificación. Para cada actividad $j$, mediante el análisis del camino crítico, obtenemos el primero y el último de los TPF de la actividad que denotamos $E F T_{j}$ y $L F T_{j}$. Representamos por $E_{j}=\left\{E F T_{j}, \ldots, L F T_{j}\right\}$, su conjunto de TPF, y por $Q_{j t}=\left\{t, \ldots, t+d_{j}-1\right\}$.

El RCPSP $/ \pi$ consiste en secuenciar las actividades de manera que se satisfagan las 
relaciones de precedencia y las restricciones de los recursos y se minimice el tiempo total de duración del proyecto o makespan.

Si definimos las variables:

$$
x_{j t}= \begin{cases}1 & \text { si la actividad } j \text { acaba en el tiempo } t \\ 0 & \text { en otro caso. }\end{cases}
$$

se puede formular como:

$$
\begin{array}{cc}
\operatorname{Min} \sum_{t \in E_{n}} t x_{n t} & \\
\text { s.t. } \quad \sum_{t \in E_{j}} x_{j t}=1 & j \in J \\
\sum_{t \in E_{i}} t x_{i t} \leq \sum_{t \in E_{j}}\left(t-d_{j}\right) x_{j t} & j \in J, i \in P_{j} \\
\sum_{j \in J} k_{j r} \sum_{t \in \Pi_{r}} \sum_{q \in Q_{j t} \cap E_{j}} x_{j q} \leq K_{r} & r \in R \\
x_{j t} \in\{0,1\} & j \in J, \quad t \in E_{j}
\end{array}
$$

La función objetivo (1) minimiza el tiempo final de la última actividad y, por tanto, la duración del proyecto. Las restricciones (2) aseguran que cada actividad acaba una sola vez. Las restricciones (3) son las de precedencia y (4) las de recursos. Nótese que a diferencia del problema con recursos renovables donde hay una restricción por cada recurso y periodo, en este problema sólo hay una restricción global para cada recurso $r \in R$. Otra característica especial de este problema es que todas las actividades deben acabar dentro del intervalo cerrado $E_{j}$ porque el conjunto $\Pi_{r}$ está definido dentro del horizonte de planificación $(0, T)$. Por tanto, no está garantizada la existencia de soluciones posibles. De hecho, Schirmer[9] ha probado que el problema de factibilidad del RCPSP $/ \pi$ es NP-completo en sentido estricto.

La formulación anterior se conoce como formulación normalizada de Böttcher et al. [3] y Schimer[9]. En sus trabajos también han considerado formulaciones alternativas pero finalmente adoptaron la normalizada por su simplicidad.

\section{El preproceso}

A través de la aplicación del preproceso se pretende alcanzar dos objetivos: El primero consiste en detectar si dado un problema concreto éste tiene o no una solución factible. En este tipo de problemas la existencia de una solución posible no está garantizada. El segundo objetivo es reducir la cantidad de recursos y de TPF de las actividades. Si se consiguen estos dos objetivos, los procedimientos de solución no perderán tiempo tratando de resolver problemas imposibles y concentrarán sus esfuerzos en los elementos significativos del problema. El preproceso que hemos desarrollado incluye varios procedimientos: 
1. Identificar los problemas triviales. Se secuencia cada actividad $j$ en su EFTj. Si esta solución cumple las restricciones de recursos, es la solución óptima.

2. Reducir el horizonte de planificación. Nosotros construimos una primera solución aplicando un algoritmo iterativo GRASP pero pudiera ser cualquier otro procedimiento constructivo. Si el makespan de esta solución, $t_{S}$, coincide con el que proporcionaría el CPM, la solución es óptima y el preproceso finaliza. En caso contrario, hacemos $T=t_{S}$. Con este nuevo $T$ se recalculan los $L F T j$ reducidos y con ellos los intervalos $E j$.

3. Filtros sobre los recursos: Eliminar los recursos ociosos y los no restrictivos. En primer lugar, eliminamos recursos ociosos, es decir, aquellos recursos $r$ que no son utilizados por ninguna actividad. Esto puede suceder si $\forall j \in J$ con $k_{r j} \neq 0 \rightarrow$ $\Pi_{r} \bigcap E j=\emptyset$.

A continuación, tratamos de detectar los recursos que no son restrictivos. Para ello calculamos para cada uno de los recursos no eliminados una cota superior de la cantidad máxima que de él se demanda. Lo hacemos resolviendo el siguiente problema lineal:

$$
\begin{aligned}
& \operatorname{Max} \quad D M D_{r}=\sum_{j=2}^{J-1} k_{j r} \sum_{t \in \Pi_{r}} \sum_{q \in Q_{j t} \cap E_{j}} x_{j q} \\
& \text { s.t. } \sum_{t=E F T_{j}}^{L F T_{j}} x_{j t}=1 \quad(1 \leq j \leq J) \\
& \sum_{m=t}^{T} x_{i m}+\sum_{s=1}^{t+d_{j}-1} x_{j s} \leq 1 \quad\left(j \leq J ; i \in P_{j} ; t \leq T\right) \\
& x_{j t} \leq 0 \quad\left(1 \leq j \leq J ; E F T_{j} \leq t \leq L F T_{j}\right)
\end{aligned}
$$

Se trata de maximizar la demanda de cada recurso (6), sujeto a que cada actividad debe acabar en un y sólo en un instante (7), se respeten las relaciones de precedencia (8), y todas las variables son no negativas. Si este máximo no supera la disponibilidad del recurso, no será restrictivo y puede eliminarse.

4. Filtros sobre las variables: Eliminación de variables. Calculamos, para cada uno de los TPF de cada actividad $j$ y cada recurso, el consumo que realizaría esa actividad si acabase en ese tiempo junto con el consumo que como mínimo realizarían el resto de actividades teniendo en cuenta que $j$ acaba en ese TPF. Si esta estimación del consumo global excede la disponibilidad del recurso, el TPF no será posible y es eliminado. Al eliminar un TPF de una actividad se comprueba la coherencia de los TPF de una actividad con los TPF de sus antecesoras y sus sucesoras. Si alguno de éstos es imposible, se suprime. Si como consecuencia de estos procesos alguna actividad se queda sin TPF el problema es imposible. 
5. Construcción de una cota inferior para la duración del problema. Después de haber disminuido las dimensiones del problema con las fases descritas previamente del preproceso, intentamos resolver el problema como si fuera un problema lineal, es decir, relajando la restricción que obliga a que las variables sean enteras. La formulación empleada es la expuesta en la sección 2. No obstante, las restricciones donde se recogen las relaciones de precedencia, serán formuladas como en el problema lineal empleado para calcular la demanda máxima de un recurso. Este proceso pretende obtener una cota inferior sobre la solución óptima del problema. Este procedimiento funciona como sigue: una vez tenemos el problema formulado tal y como hemos comentado, lo resolvemos aplicando un código de programación lineal, CPLEX. El valor óptimo obtenido para el problema lineal, opl $l_{1}$, redondeado por exceso, es una cota mínima para el valor óptimo del final de la última actividad, J, del proyecto. A continuación, pasamos a eliminar todos aquellos posibles tiempos de finalización de la última actividad que sean menores que $\left\ulcorner o p l_{1}\right\urcorner$. Por tanto, las variables relacionadas con esos tiempos se anularán, es decir, $x_{J t}:=0$ para todos los $t<\left\ulcorner o p l_{1}\right\urcorner$. Si conseguimos eliminar algún tiempo, de nuevo resolvemos la versión lineal del problema modificado. Si obtenemos una nueva solución $o p l_{2}$ estrictamente mayor que $o p l_{1}$, volvemos a suprimir las variables relacionadas con el final de la última actividad. Este proceso se repetirá hasta que ningún tiempo pueda ser eliminado. Tras agotar el proceso el último valor encontrado redondeado por exceso, Cota_Lineal $=\left\ulcorner o p l_{m}\right\urcorner$, es una cota inferior que, en ocasiones, nos va a servir para saber si una metaheurística concreta ha encontrado o no la solución óptima del problema.

Para acabar esta descripción del preproceso, en el que se han ido eliminando recursos y variables, hemos de hacer notar que, además de identificar problemas no posibles, es útil para poder identificar la optimalidad de la solución de valor $F_{J}$ obtenida en la fase 2. ¿Cómo podemos saber si $F_{J}$ es el óptimo?. Para ello compararemos este valor con las siguientes tres cotas:

(a) Si $F_{J}=$ valor proporcionado por CPM entonces $F_{J}$ es óptimo. Para que esto se cumpla, la composición de la solución proporcionada por el CPM y la de la solución inicial no tiene que ser igual necesariamente. El final de todas las actividades, a excepción de la última, puede ser diferente y sólo el de la última actividad debe coincidir en ambos casos.

(b) Si el final que la última actividad tenía asignada en la solución que inicialmente construímos es igual al mínimo tiempo de finalización que ha quedado tras aplicar todos los procesos de filtrado expuestos, entonces, esa solución es la óptima.

(c) Si las dos cotas anteriores no prueban la optimalidad del problema, se calcula la Cota_Lineal tal y como hemos comentado. Si $F_{J}=$ Cota_Lineal, entonces $F_{J}$ es óptimo.

Todos los pasos del preproceso expuestos en esta sección pueden encontrarse más detalladamente en $[1,2]$. 
194 R. Alvarez - E. Crespo - J.M. Tamarit - F. Villa Rev.Mate.Teor.Aplic. (2009) 16(1)

En la figura 1 se muestra un esquema general del funcionamiento del preproceso descrito.

\section{Resultados computacionales}

\subsection{Instancias para las pruebas}

Böttcher et al.[3] generaron un primer conjunto de instancias para pruebas. Tomaron como punto de partida PROGEN 2 [5], un generador de instancias para el clásico RCPSP con recursos renovables, modificaron y agrandaron el conjunto de parámetros y generaron un conjunto de 2160 instancias con 10 actividades no ficticias, 10 réplicas para cada una de las 216 combinaciones de valores de los parámetros. Como la mayoría de los problemas fueron imposibles restringieron los valores de los parámetros a las 25 combinaciones más prometedoras y generaron 250 instancias con 15, 20, 25 y 30 actividades operativas, manteniendo siempre la cantidad de 30 recursos.

Más recientemente, Schirmer[9] desarrolló PROGEN 3, una extensión de PROGEN 2, y generó 960 instancias de tamaños 10, 20, 30, y 40, con 30 recursos. La mayoría tenían solución factible, aunque unos pocos eran infactibles y algunos se etiquetaron como no decididos porque en el tiempo límite fijado para el algoritmo de branch and bound de Böttcher et al.[3] éste fue incapaz de obtener una solución pero tampoco pudo probar su infactibilidad.

\subsection{Resultados del preproceso}

Los procedimientos del preproceso mostrados en la sección 3 son los que se han aplicado a los problemas de Böttcher et al.[3] de 10, 15, 20, 25 y 30 actividades que estaban disponibles solicitándolos a los autores. En las Tablas 1, 2 y 3 aparecen diferentes aspectos de los resultados. La Tabla 1 muestra los logros del preproceso para determinar el estatus de los problemas. Hemos señalado como posibles aquellas instancias para las que el preproceso encuentra una solución y como imposibles aquellas que el preproceso puede demostrar que no tienen solución. Cuando no ha sido posible determinar dicho estatus los hemos marcado como no decididos. En resumen, podemos decir que nuestro preproceso es muy eficiente para determinar el estatus de una instancia dada.

\begin{tabular}{l|rrrrr}
\hline \hline & $\mathrm{n}=10$ & $\mathrm{n}=15$ & $\mathrm{n}=20$ & $\mathrm{n}=25$ & $\mathrm{n}=30$ \\
\hline Problemas & 2160 & 250 & 250 & 250 & 250 \\
Detectados como imposibles & 1205 & 16 & 17 & 12 & 8 \\
Detectados como possibles & 879 & 233 & 231 & 236 & 239 \\
No decididos & 76 & 1 & 2 & 2 & 3 \\
Categoría real & Imposibles & Posible & No decididos & Imposibles & No decididos \\
\hline \hline
\end{tabular}

Tabla 1: Problemas de Böttcher et al. - Determinación del estatus.

La última línea de la Tabla 1 muestra el estatus que hemos sido capaces de determinar 


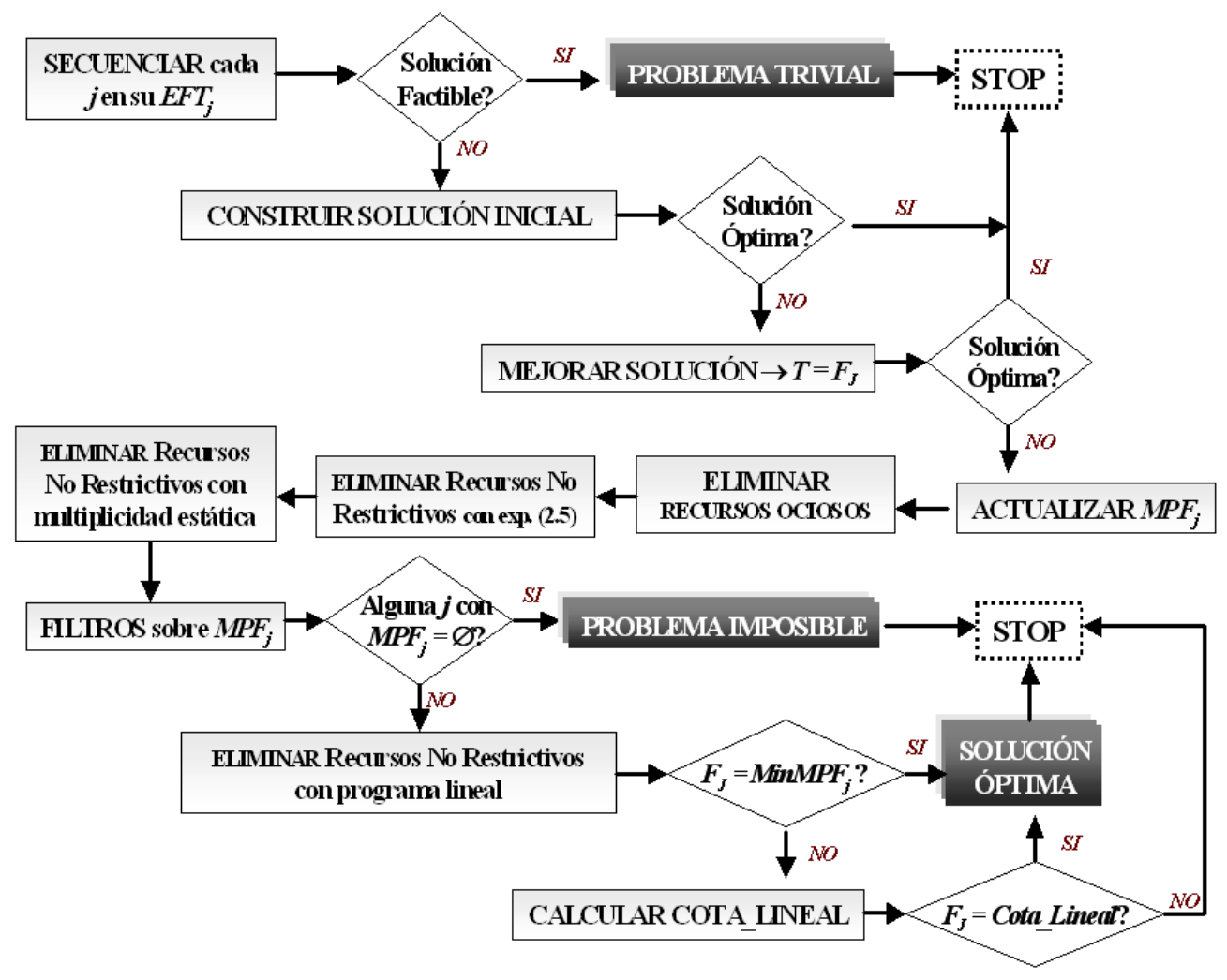

Figura 1: Esquema general del preproceso.

para los problemas que quedaban por decidir después del preproceso. Para ello usamos CPLEX con una formulación entera del problema adaptada a partir de la expuesta en la Sección 2. Incluso en este caso para 2 instancias de tamaño 20 y 3 de tamaño 30 ha sido imposible determinar su estatus, aunque suponemos que serán imposibles.

La Tabla 2 muestra las soluciones óptimas que obtiene el preproceso. En esta tabla no se han incluido los problemas que quedaban como no decididos porque es muy improbable que tengan solución. Para más del $70 \%$ de las instancias las técnicas del preproceso han obtenido los óptimos.

La Tabla 3 presenta la reducción de recursos y variables conseguida para aquellos problemas no resueltos por el preproceso. Las rápidas técnicas del preproceso reducen significativamente la cantidad de recursos a considerar y, todavía más importante, la cantidad de valores posibles de las variables de decisión.

Los resultados obtenidos para los problemas de prueba generados por Schirmer[9] son de calidad similar. La Tabla 4 muestra los resultados del preproceso al determinar el status de los problemas y proporcionando soluciones óptimas para la mayoría de ellos. Hay que resaltar que los status que quedaban pendientes en el libro de Schirmer[9] aquí han sido determinados. De hecho se prueba que todos ellos son factibles excepto para 5 instancias 


\begin{tabular}{l|rrrrr}
\hline \hline & $\mathrm{n}=10$ & $\mathrm{n}=15$ & $\mathrm{n}=20$ & $\mathrm{n}=25$ & $\mathrm{n}=30$ \\
\hline Problemas & 2160 & 250 & 250 & 250 & 250 \\
Problemas posibles & 879 & 234 & 231 & 236 & 239 \\
Problemas triviales & 52 & 46 & 85 & 114 & 128 \\
Óptimos obtenidos por el preproceso & 689 & 148 & 117 & 90 & 80 \\
Problemas restantes & 138 & 40 & 29 & 32 & 31 \\
\hline \hline
\end{tabular}

Tabla 2: Problemas de Böttcher et al. - Soluciones óptimas identificadas en el preproceso.

\begin{tabular}{l|rrrrr}
\hline \hline & $\mathrm{n}=10$ & $\mathrm{n}=15$ & $\mathrm{n}=20$ & $\mathrm{n}=25$ & $\mathrm{n}=30$ \\
Problemas & 138 & 40 & 29 & 32 & 31 \\
\hline Recursos iniciales & 30 & 30 & 30 & 30 & 30 \\
Recursos restantes (media) & $20(67 \%)$ & $17(57 \%)$ & $24(80 \%)$ & $24(80 \%)$ & $24(80 \%)$ \\
Variables iniciales (media) & 93 & 264 & 564 & 885 & 1298 \\
Variables restantes (media) & $55(59 \%)$ & $139(53 \%)$ & $362(64 \%)$ & $624(71 \%)$ & $872(67 \%)$ \\
\hline \hline
\end{tabular}

Tabla 3: Problemas de Böttcher et al. - Reducción de recursos y variables.

de tamaño 10 que son imposibles como ya conjeturó Schirmer. Para más del $60 \%$ de los problemas no triviales el preproceso es capaz de obtener una solución óptima probada.

\begin{tabular}{l|rrrrr}
\hline \hline & $\mathrm{n}=10$ & $\mathrm{n}=20$ & $\mathrm{n}=30$ & $\mathrm{n}=40$ & $\mathrm{n}=60$ \\
\hline Problemas & 951 & 960 & 960 & 960 & 960 \\
Problemas posibles & 946 & 960 & 960 & 960 & 960 \\
Problemas triviales & 143 & 395 & 507 & 574 & 614 \\
Óptimos obtenidos por el preproceso & 502 & 341 & 291 & 221 & 202 \\
Problemas restantes & 301 & 224 & 162 & 165 & 144 \\
\hline \hline
\end{tabular}

Tabla 4: Problemas de Schirmer - Soluciones óptimas identificadas en el preproceso.

La Tabla 5 presenta las reducciones que el preproceso obtiene en el número de recursos y variables.

\section{Conclusiones}

Hemos estudiado una generalización del problema clásico de secuenciación de proyectos con limitación de recursos. Hemos considerado un tipo de recursos relativamente nuevo, los recursos parcialmente renovables, donde la disponibilidad de un recursos está asociada a un conjunto dado de periodos y las actividades sólo lo consumen si son procesadas en dichos periodos. Este tipo de recursos pueden considerarse como una generalización tanto de lo recursos renovables como de los no renovables, pero su principal interés proviene de su utilidad para modelizar situaciones que aparecen en los problemas de turnos, horarios 


\begin{tabular}{l|rrrrr}
\hline \hline & $\mathrm{n}=10$ & $\mathrm{n}=20$ & $\mathrm{n}=30$ & $\mathrm{n}=40$ & $\mathrm{n}=60$ \\
Problemas & 301 & 224 & 162 & 165 & 144 \\
\hline Recursos iniciales & 30 & 30 & 30 & 30 & 30 \\
Recursos restantes (media) & $15(50 \%)$ & $15(50 \%)$ & $18(60 \%)$ & $18(60 \%)$ & $20(67 \%)$ \\
Variables iniciales(media) & 211 & 971 & 2281 & 4256 & 9962 \\
Variables restantes (media) & $103(49 \%)$ & $338(35 \%)$ & $718(31 \%)$ & $1188(28 \%)$ & $2616(26 \%)$ \\
\hline \hline
\end{tabular}

Tabla 5: Problemas de Schirmer - Reducción de recursos y variables.

de trabajo y calendarios que pueden ser formulados como problemas de secuenciación de proyectos.

Hemos desarrollado diferentes técnicas de preproceso que ayudan a determinar la existencia de soluciones posibles y a reducir la cantidad de variables y restricciones. El preproceso, que ha sido probado en dos conjuntos de instancias provinientes de la literatura, ha sido capaz de determinar el status de factibilidad de muchas de las instancias que hasta el momento estaba sin determinar y de resolver óptimamente la mayoría de las instancias posibles.

Estamos convencidos que las técnicas de preproceso aquí desarrolladas deberían ser usadas por cualquier procedimiento de solución, exacto o heurístico, aplicado a este problema.

\section{Referencias}

[1] Alvarez-Valdés, R.; Crespo, E.; Tamarit, J.M.; Villa, F. (2006) "GRASP and path relinking for project scheduling under partially renewable resources", European Journal of Operational Research, en prensa.

[2] Alvarez-Valdés, R.; Crespo, E.; Tamarit, J.M.; Villa, F. (2006) "A scatter search algorithm for project scheduling under partially renewable resources", Journal of Heuristics 12: 95-113.

[3] Böttcher, J.; Drexl, A.; Kolisch, R.; Salewski, F. (1999) "Project scheduling under partially renewable resource constraints", Management Science 45: 544-559.

[4] Demeulemeester, E.L.; Herroelen, W.S. (2002) Project Scheduling: A Research Handbook. Kluwer Academic Publishers, Boston.

[5] Kolisch, R.; Sprecher, A.; Drexl, A. (1995) "Characterization and generation of a general class of resource-constrained project scheduling problems", Management Science 41: $1693-1703$.

[6] Mellentien, C.; Schwindt, C.; Trautmann, N. (2004) "Scheduling the factory pick-up of new cars", OR Spectrum, in press. 
198 R. Alvarez - E. Crespo - J.M. TAmarit - F. Villa Rev.Mate.Teor.Aplic. (2009) 16(1)

[7] Neumann, K.; Schwindt, C.; Trautmann, N. (2002) "Advanced production scheduling for batch plants in process industries", OR Spectrum 24: 251-279.

[8] Neumann, K.; Schwindt, C.; Trautmann, N. (2004) "Scheduling of continuous and discontinuous material flows with intermediate storage restrictions", European Journal of Operational Research, in press.

[9] Schirmer, A. (2000) Project Scheduling with Scarce Resources. Verlag Dr. Kovac, Hamburg.

[10] Schwindt, C.; Trautmann, N. (2000) "Scheduling the production of rolling ingots: industrial context, model and solution method", International Transactions in Operations Research 10: 547-563. 